\title{
4081 Times Increase in Atmospheric Humidity Capture Capacity via Confinement of 2D Nanosheets in 3D Matrix
}

\section{Lan Zhang} \\ Southeast University

\section{Shu-Hua Ma} \\ Southeast University \\ Hui Dong \\ Southeast University \\ Feng-Lian Zeng \\ Southeast University

\section{Ya-Chen Zou} \\ Southeast University

\section{$\mathrm{Ke}-\mathrm{Xin} \mathrm{Xu}$} \\ Southeast University
}

Yang-Hui Luo ( $\sim$ peluoyh@sina.com )

Southeast University https://orcid.org/0000-0002-5555-2510

\section{Research Article}

Keywords: Intelligent gel, Humidity capture, In-situ liquefaction, Confinement, 2D materials

Posted Date: December 17th, 2021

DOI: https://doi.org/10.21203/rs.3.rs-1147384/v1

License: (9) (i) This work is licensed under a Creative Commons Attribution 4.0 International License. Read Full License 


\section{Abstract}

Atmospheric humidity capture is urgently needed in humidity-related process. Most of the traditional technology suffers the defects of large energy consumption, low capture capacity, etc. Here, an intelligent gel (IG), which was composed of super hygroscopic materials (SHM, for humidity detection, spontaneous capture and in-situ liquefaction), hydrophilic polymer PEG-6000 (polyethylene glycol, for humidity storage), and hydrophobic polymer PVDF (poly(vinylidene-fluoride), for fast water release), is shown. Based on such kind of integration, effective color responsive humidity detection, spontaneous humidity capture and in-situ liquefaction, under ambient conditions, have been achieved. The synergistic effects between two polymers have given birth to a 3D polymer framework that can shrink upon heating and swelling upon solvent immersion, which then act as a versatile matrix that dispersed evenly the 2D SHM into atomically scale. As a consequence, approximate 4081 times increase in working capacity than the individual SHM has been observed. Being an effective way to manipulate atmospheric humidity at the device level, the present work may open new avenue for next-generational water management systems.

\section{Introduction}

Nanocomposites, the multi-phase materials that made of two or more components with different properties, are of importance owing to the improved performance. (Ayala et al., 2019; Zhang et al., 2021; El-Mehalmey et al., 2020; Tan et al., 2019; Unnikrishnan et al., 2021) At present, the research focus for nanocomposites was on how to prepare macro-sized composite by assembling nanomaterials with others, while keeping the properties the former. (Zhong et al., 2021; Kitao et al., 2017; Keinath et al., 2021; Uemura et al., 2005) Metal-organic frameworks (MOFs), for example, the nanoscale MOFs have limited their application in various fields due to their poor stability and weak mechanical strength. Assemble of nanoscale MOFs with proper functional materials, such as polymers, inorganic salts, graphene and magnetic nanoparticles, have been proved to give birth to promising synergistic effects: on the one hand, retain of properties of MOFs; on the other hand, overcome the shortcomings. (Liu et al., 2020; Xu et al., 2020; Huo et al., 2019; Mehdinia et al., 2018; Troyano et al., 2018)

It is widely needed to capture atmospheric humidity in the humidity-related process. The traditional technologies, such as condensation or desiccant, suffers defects of large energy consumption, low capture capacity, etc. (Wang et al., 2021; Zain et al., 2021; Lou et al., 2021) Porous materials based on hydration can be used as effective humidity collection in atmosphere. A typical example was the MOFs that can actively capture water vapor in the atmosphere at relatively low humidity and then release water via sunlight irradiation. (Hanikel et al., 2019) For example, Yaghi, et al. (Hanikel et al., 2020) have reported in 2017 that the MOF-801 can capture potable pure water from dry desert air, with no additional energy input except for natural sunlight. Generally, a kilogram of MOF-801 can harvest 2.8 liters of purified water per day in desert environment with a relative humidity of as low as $20 \%$. This attractive result was mainly due to the sponge like chemical structure of MOFs. However, one the one hand, for most of the MOFs, their adsorption saturation can be achieved in a short time, which makes the relatively low moisture absorption capacity even if the relative humidity is higher (greater than $50 \% \mathrm{RH}$ ); on the other hand, some 
porous MOFs have disadvantages of poor stability and weak mechanical strength, which have limited their application in various fields to some extent. (Kim et al., 2019; Kim et al., 2018; Liu et al., 2020; Mulchandani et al., 2020; Xu and Yaghi, 2020) Based on the above discussions, the combination of water harvesting MOFs with functional polymers which possess the merits of high strength, good hydrophilicity, easy of processing and flexible, may act as a promising strategy for the development of super materials for water harvesting and humidity detection.

In our previously work, (Luo et al., 2021) a two-dimensional (2D) super hygroscopic materials (SHM) 2D$\mathrm{Co} / 2 \mathrm{D}-\mathrm{Zn}\left(2 \mathrm{D}-\mathrm{Co}=\left[\mathrm{Co}(\mathrm{NCS})_{2}(\mathrm{pyz})_{2}\right], 2 \mathrm{D}-\mathrm{Zn}=\left[\mathrm{Zn}(\mathrm{NCS})_{2}(\mathrm{pyz})_{2}\right]\right.$, pyz $=$ pyrazine $)$, which shown capable of adsorbing atmospheric water at over $658 \%$ of its own weight, has been prepared. However, the reunite of SHM during the adsorption process has obstructed its widespread application. Here in this work, we present an intelligent gel (IG), which synergistically combines 2D SHM, hydrophilic polymer PEG-6000 (polyethylene glycol) and hydrophobic polymer PVDF (poly(vinylidene-fluoride), that can solve the abovementioned dilemma. Among such kind of integration, the hydrophobic PVDF, which possess good stability and heat resistance due to its high dissociation C-F bond energy, (Yilmaz et al., 2019, Wang et al., 2021; Badatya et al., 2021) has acted as the main frame of IG and responsible for fast water release; the hydrophilic PEG, which was added to improve the affinity of PVDF to water, was responsible for humidity storage in IG; the SHM, which has shown ultra-high water adsorption capacity and natural sunlight triggered water release under ambient conditions, as well as remarkable humidity-dependent colorchange, was responsible for humidity detection, spontaneous capture and in-situ liquefaction on IG. Interestingly, the PVDF and PEG have formed a three-dimensional (3D) polymer framework that can shrink upon heating and swelling upon solvent immersion, which has served as a versatile matrix to dispersed evenly the 2D SHM into atomically scale, with optimal weight ratio of SHM:PVDF $=30 \%$. As a consequence, effective color responsive humidity detection, spontaneous humidity capture (with highest capture capacity of $0.77 \mathrm{~g} / \mathrm{g}(\mathrm{IG})$ or $26855 \mathrm{~g} / \mathrm{g}(\mathrm{SHM})$ ) and in-situ liquefaction, under ambient conditions, have been achieved with IG. Compared with the individual SHM with highest capture capacity of $6.58 \mathrm{~g} / \mathrm{g}$, the incorporation into IG has increase in approximate 4081 times the working capacity of SHM, which thus providing an effective strategy to manipulate atmospheric humidity at the device level, and may open new avenue for next-generational water management systems.

\section{Results And Discussion}

The preparation strategy and working principles of IG were illustrated in Figure 1. The yellow-colored matrix (Figure 1a) was composed by gelation of PVDF (Figure 1b) and PEG (Figure 1c). While, the bluecolored SHM (Figure 1d), which has shown fluffy and disordered motifs (Figure 1e), was prepared via insitu exfoliation (Figure 1f) of hetero-layered 2D MOFs $\left[\mathrm{Co}(\mathrm{NCS})_{2}(\mathrm{pyz})_{2}\right]$ and $\left[\mathrm{Zn}(\mathrm{NCS})_{2}(\mathrm{pyz})_{2}\right]$ (Figure 1g, Figure S1 and S2). It should be note that, SHM can be well dispersed in ethanol solution to give a colloidal suspension with a significant Tyndall effect (Figure 1h), suggesting the atomically thin 2D nature (Figure S3). In addition, the key aspects of SHM for humidity capture lies on the larger 2D surfaces area, as well as the high polarity of pyrazine linker and NCS ligand. Hence, the more exposure of the 2D 
surfaces, the higher working capacity of SHM. At this point, the yellow-colored blank gel can act as versatile bracket to dispersed evenly the SHM into atomically scale (Figure S4). As a consequence, the resulted IG (Figure 1i) was highly sensitive to atmospheric humidity, which can capture humidity effectively with continuously color-change (Figure 1j) and in-situ liquefaction (Figure 1k), and this process can be recyclable, providing a pretty good model for atmospheric humidity manipulation at the device level.

The successfully application of IG was highly dependent on the flexibility of the polymer framework, the good compatibility with SHM and appropriate flex channel for water delivery are required. To verify these aspects, the flexibility of IG with weight ratio of SHM:PVDF $=3 \%$ was investigated. A sky-blue colored cubic of IG with side-length of $6 \mathrm{~mm}$ was prepared (Figure 2a), upon exposure to ambient air, continuous water adsorption was visible to the naked eye, with the color of the cubic has changed into grey at $10 \mathrm{~min}$ (Figure S5) and finally into light-pink (Figure 2b). However, no increase in side-length can be observed,

suggesting a good adaptability of IG to water. More importantly, Raman measurements have demonstrated that upon water capture, the spectrum of SHM have been disappear with only the spectrum of water presented (Figure S6), suggesting that the water molecules were adsorbed on the 2D surfaces of SHM. While upon heating under $40^{\circ} \mathrm{C}$ for $1 \mathrm{~h}$ with continuous water release, the light-pink colored cubic has shrinking into a blue-colored cubic with side-length of about $4 \mathrm{~mm}$, demonstrating the good flexibility of the polymer framework. The SEM images of IG under the above-mentioned three different state were then investigated. For the as-prepared state, channels with different sizes (average diameter of about 5 $\mu \mathrm{m}$ ) were fill the whole gel (Figure $2 \mathrm{~d}, \mathrm{e}$ ), which are expected to promote greatly the efficiency of water adsorption on IG. While upon water adsorption, the sizes of channels were expanded about 8 times, with average diameter to be about $40 \mu \mathrm{m}$ (Figure $2 \mathrm{f}, \mathrm{g}$ ), providing a wide and unobstructed passage for shuttle of water molecules. It should be note, the expand of channels did not induced the increase the whole volume of gel, suggesting the intelligent nature of this gel with superior adaptability to water.

At this point, water release must lead to shrinking of the channels on IG, as expected, the diameter of the channel has decreased to be about $1 \mu \mathrm{m}$ (Figure $2 \mathrm{~h}$, i). Note again, this kind of 40 times shrinking in channels has only induced about 3.4 times decrease of the gel volume, further demonstrating the intelligence of the gel with superior adaptability to water.

For practical application, resume of the shrinking gel is need. On considering the cross-linking structure, immersion in benign solvent is expected to swell the shrinking gel. We first choose aqueous solution, for the sky-blue-colored samples, the immersion in water have given birth to a white cubic (Figure 3a), however, no increase in volume can be observed even immersed for $12 \mathrm{~h}$ (Figure 3b), suggesting that water is the bad solvent for IG, then the in-situ liquefaction of the adsorbed water in IG can be expected. While for the samples after heated under $40^{\circ} \mathrm{C}$ for $40 \mathrm{~min}$ (with side-length of $4 \mathrm{~mm}$ ), the immersion in DMF solution has shown continuously fast increase in side-length of the cubic (Figure 3c), from the begin $4 \mathrm{~mm}$ to $5.5 \mathrm{~mm}$ at $5 \mathrm{~min}$, then $6.5 \mathrm{~mm}$ at $10 \mathrm{~min}$, and finally to $7 \mathrm{~mm}$ at $50 \mathrm{~min}$. Interestingly, for the swelling cubic with side-length of $7 \mathrm{~mm}$, the blue-colored SHM was distributed in the inner space within 5 
$\mathrm{mm}$, almost similar with the as-prepared state. That is, the swelling process did not affect the distribution position of SHM, suggesting the well stability of IG during the process of water adsorption and release. In addition, this kind of IG has shown a much fast swelling process than the shrinking (Figure 3d), the former has reached a 3.5 ratio within 50 min, while the latter got a 0.7 ratio at $100 \mathrm{~min}$, providing a 10 times disparity in efficiency. One thing should be stressed is that, heating under $40{ }^{\circ} \mathrm{C}$ for more than $2 \mathrm{~h}$ (with side-length of $3.5 \mathrm{~mm}$ ) will destroy the elasticity of IG, as no swelling of the shrinking gel can be observed even been immersed in DMF for more than 50 min (Figure 3e), accompanied by a 3 time decrease in water adsorption capacity (Figure 3f).

The above-mentioned results have demonstrated the intelligent, elastic and stable aspects of the polymer framework, then the optimal doping of SHM in IG needs to be explored, for the purpose of fully expose the 2D surface as well as high polarity pyrazine linker and NCS ligands, to get the optimum performance of IG. The doping of SHM in IG was evaluated by weight ratio of SHM:PVDF, which have been set as 3, 10, $20,30,40,50$, and $70 \%$. As have shown in Figure 4 and Figure S7, for all of the ratios, fast water adsorption, continuously dramatic color change, as well as continuously in-situ liquefaction, all have been observed within $3 \mathrm{~h}$ upon exposure the gel cubic to ambient atmosphere. However, the difference still remains (Figure $4 a)$, that is: for ratios less than $10 \%(3 \%)$ and larger than $40 \%(40,50,70 \%)$, the color change were all not obviously, the former can be attribute to the too small amounts of SHM, while the latter was because of too much amounts of SHM that were accumulated over dense, which adverse to the fully exposure the 2D surfaces as well as high polarity pyrazine linker and NCS ligands.

Actually, for the cubic gels with SHM: PVDF weight ratio of 10, 20 and $30 \%$, the fast water adsorption, the sensitive and huge contrast of colors induced by adsorption capacities, the efficient in-situ liquefaction with no overflow of SHM (Figure 4a), all have demonstrated that this kind of intelligent gel has shown promising applications in atmospheric humidity detection, spontaneous capture and in-situ liquefaction. While for these three valid ratios, the time-dependent water uptake profile has suggested that the cubic gel with SHM: PVDF weight ratio of $30 \%$ has shown the largest water adsorption capacity, reaching a value of $0.77 \mathrm{~g} / \mathrm{g}(\mathrm{IG})$ at $1500 \mathrm{~min}$ (Figure 4b), which was followed successively by ratio of 50, 20, 10 and $40 \%$. One thing should be stressed is that, the water adsorption of IG was only owe to SHM. Hence, the water adsorption capacity based on the amount of SHM deserves to be investigated. Figure 4c has shown the SHM-based water adsorption capacity, where, the ratio of $30 \%$ have displayed a value as high as $26856 \mathrm{~g} / \mathrm{g}(\mathrm{SHM})$, which was substantially larger than the value of 21485, 18979, 14925, and 11976 $\mathrm{g} / \mathrm{g}(\mathrm{SHM})$, for ratio of $20,10,40$, and $50 \%$, respectively. Compared with the water adsorption capacity of $6.58 \mathrm{~g} / \mathrm{g}(\mathrm{SHM})$ for the powdered state SHM, the present results have provide the following three beneficial aspects: (1) The integration into IG has provide a practical model for application of SHM; (2) The doping of SHM into polymer framework, with any ratios, have improved significantly the performance of SHM (3) The optimal doping ratio is SHM:PVDF $=30 \%$, which has get a 4081 times increase in working capacity than the individual SHM.

\section{Experimental Implication}


The subtle integration of super hygroscopic material (SHM), hydrophilic polymer (PEG-6000), and hydrophobic polymer (PVDF), to construct an intelligent gel (IG) for practical atmospheric humidity detection, spontaneous capture and in-situ liquefaction, under ambient conditions, have been demonstrated. Within such a design strategy, the division of labor was clear-cut for each part: (1) SHM was responsible for fast water adsorption and in-situ liquefaction; (2) PEG-6000 was act as a modifier to assist PVDF to form porous polymer framework for water storage; (3) PVDF was the main framework of IG, which form crisscross channels for water molecules adsorption and release. This synergistic effect involves three parts has improved 4081 times the working capacity of SHM, which are expected to open new avenue for next-generational water management systems.

\section{Materials And Methods}

Preparation of IG: Step 1: $0.8 \mathrm{~g}$ of PEG-6000 were dispersed in $25 \mathrm{ml}$ of dimethylformamide (DMF) to give a clear solution, then $3 \mathrm{~g}$ of PVDF were add gradually under continuously stirring. After stirred for $2 \mathrm{~h}$, the mixture became clear and transparent, which was then defoam under room temperature for $1 \mathrm{~h}$.

Step 2: To the above resulted mixtures, 0.3g (SHM: PVDF = 10\%) / 0.6g (SHM: PVDF $=20 \%) / 0.9 \mathrm{~g}(\mathrm{SHM}$ : PVDF $=30 \%) / 1.2 \mathrm{~g}($ SHM: PVDF $=40 \%) / 1.5 \mathrm{~g}(\mathrm{SHM}:$ PVDF $=50 \%) / 2.1 \mathrm{~g}(\mathrm{SHM}:$ PVDF $=70 \%))$ SHM was added, which was followed by ultrasonic for $1 \mathrm{~h}$ for fully dispersion of SHM in polymer framework. After that, the mixtures were transferred to the oil bath and stirred at $80{ }^{\circ} \mathrm{C}$ for $12 \mathrm{~h}$, to agitated uniformly of the gel solution. Finally, the gel solution was cooled to room temperature and standing for at least $24 \mathrm{~h}$, and followed by pouring into a clean square mold to get cubic IG.

\section{Atmospheric Water Harvesting}

Under the ambient conditions, cubic IG was put on a glass sheet and exposed to atmosphere. The adsorption rates and adsorption capacities of IG were investigated by weighing the glass sheet along with cubic IG by an electronic balance at the certain time interval.

\section{Declarations}

\section{Acknowledgements}

This research was supported by the Natural Science Foundation of China (Grant No. 21701023), Natural Science Foundation of Jiangsu Province (Grant No. BK20170660), Zhishan Youth Scholar Program of SEU and PAPD of Jiangsu Higher Education Institutions.

\section{References}

1. Ayala S, Bentz KC, Cohen SM (2019) Block co-poly-MOFs: morphology control of polymer-MOF hybrid materials. Chem Sci 10:1746-1753 
2. Badatya S, Bharti DK, Srivastava AK, Gupta MK (2021) Solution processed high performance piezoelectric eggshell membrane - PVDF layer composite nanogenerator via tuning the interfacial polarization. J Alloy Compd 863:158406

3. El-Mehalmey WA, Safwat Y, Bassyouni M, Alkordi MH (2020) Strong Interplay between Polymer Surface Charge and MOF Cage Chemistry in Mixed-Matrix Membrane for Water Treatment Applications. ACS Appl Mater Interfaces 12:27625-27631

4. Hanikel N, Prevot MS, Fathieh F, Kapustin EA, Lyu H, Wang H, Diercks NJ, Glover TG, Yaghi OM (2019) Rapid Cycling and Exceptional Yield in a Metal-Organic Framework Water Harvester. ACS Cent Sci 5:1699-1706

5. Hanikel N, Prevot MS, Yaghi OM (2020) MOF water harvesters. Nat Nanotechnol 15:348-355

6. Huo JB, Xu L, Chen X, Zhang Y, Yang JCE, Yuan B, Fu ML (2019) Direct epitaxial synthesis of magnetic Fe304@UiO-66 composite for efficient removal of arsenate from water. Micropor Mesopor Mater 276:68-75

7. Keinath MC, Davidian A, Timoshevskiy V, Timoshevskaya N, Gall JG (2021) Characterization of axolotl lampbrush chromosomes by fluorescence in situ hybridization and immunostaining. Exp Cell Res 40:112523

8. Kim B, Na J, Lim H, Kim Y, Kim J, Kim E (2019) Robust High Thermoelectric Harvesting Under a SelfHumidifying Bilayer of Metal Organic Framework and Hydrogel Layer. Adv Funct Mater 29:1807549

9. Kim H, Rao SR, Kapustin EA, Zhao L, Yang S, Yaghi OM (2018) Adsorption-based atmospheric water harvesting device for arid climates. Nat Commun 9:1191

10. Kitao T, Zhang Y, Kitagawa S, Wang B, Uemura T (2017) Hybridization of MOFs and polymers. Chem Soc Rev 46:3108-3133

11. Liu L, Li Z, Liu Y, Zhang X (2020) Recent advances in film-based fluorescence sensing. Sci ChinaChem 50:39-69

12. Liu X, Wang X, Kapteijn F (2020) Water and Metal-Organic Frameworks: From Interaction toward Utilization. Chem Rev 120:8303-8377

13. Lou C, Li Z, Yang C, Liu X, Zheng W, Zhang J (2021) Rational design of ordered porous SnO2/ZrO2 thin films for fast and selective triethylamine detection with humidity resistance. Sensor Actuat BChem 333:125972

14. Luo Y-H, Wang C, Ma S-H, Jin X-W, Zou Y-C, Xu K-X, Fang W-X, Zhang L, Dong H (2021) Humidity reduction by using hetero-layered metal-organic framework nanosheet composites as hygroscopic materials. Environ. Sci.: Nano, DOI: 10.1039/d1en00617g

15. Mehdinia A, Jahedi Vaighan D, Jabbari A (2018) Cation Exchange Superparamagnetic Al-Based Metal Organic Framework (Fe304/MIL-96(Al)) for High Efficient Removal of Pb(II) from Aqueous Solutions. ACS Sustain Chem Eng 6:3176-3186

16. Mulchandani A, Westerhoff P, Westerhoff P (2020) Geospatial Climatic Factors Influence Water Production of Solar Desiccant Driven Atmospheric Water Capture Devices. Environ Sci Technol $54: 8310-8322$ 
17. Tan Y, Meng H, Zhang X (2019) Removal of Organic Dyes and Heavy Metal lons by Functionalized MOFs and MOFs/Polymer Composite Membranes. Prog Chem 31:980-995

18. Troyano J, Carne-Sanchez A, Perez-Carvajal J, Leon-Reina L, Imaz I, Cabeza A, Maspoch D (2018) A Self-Folding Polymer Film Based on Swelling Metal-Organic Frameworks. Angew Chem Int Edit 57:15420-15424

19. Uemura T, Kitagawa K, Horike S, Kawamura T, Kitagawa S, Mizuno M, Endo K (2005) Radical polymerisation of styrene in porous coordination polymers. Chem Commun 48:5968-5970

20. Unnikrishnan V, Zabihi O, Ahmadi M, Li Q, Blanchard P, Kiziltas A, Naebe M (2021) Metal-organic framework structure-property relationships for high-performance multifunctional polymer nanocomposite applications. J Mater Chem A 9:4348-4378

21. Wang F, Wang B, Zhang X, Lu M, Zhang Y, Sun C, Peng W (2021) High Sensitivity Humidity Detection Based on Functional GO/MWCNTs Hybrid Nano-Materials Coated Titled Fiber Bragg Grating. Nanomaterials 11, 11051134

22. Wang $H$, Zhang J, Wang D, Wang Z, Chen Y, Feng X (2021) Flexible triphase enzyme electrode based on hydrophobic porous PVDF membrane for high-performance bioassays. Biosens Bioelectron 183:113201

23. Xu J, Li T, Chao J, Wu S, Yan T, Li W, Cao B, Wang R (2020) Efficient Solar-Driven Water Harvesting from Arid Air with Metal-Organic Frameworks Modified by Hygroscopic Salt. Angew Chem Int Edit 59:5202-5210

24. Xu W, Yaghi OM (2020) Metal-Organic Frameworks for Water Harvesting from Air, Anywhere, Anytime. ACS Cent Sci 6:1348-1354

25. Yilmaz G, Peh SB, Zhao D, Ho GW (2019) Atomic- and Molecular-Level Design of Functional MetalOrganic Frameworks (MOFs) and Derivatives for Energy and Environmental Applications. Adv Sci 6:1901129

26. Zain HA, Batumalay M, Rahim A, Yasin HR, Harun M, S.W (2021) Effect of agarose concentration on coated micro-bottle resonators for humidity detection. Microw Opt Techn Let 63:1826-1831

27. Zhang G, Zhang Y, Yang J, Li S, Guo W (2021) Preparation and Characterization of Wood-Plastic Nanocomposites Based on Acrylonitrile-Butadiene-Styrene. J Nanosci Nanotechnol 21:4840-4845

28. Zhong M, Kong L, Zhao K, Zhang YH, Li N, Bu XH (2021) Recent Progress of Nanoscale MetalOrganic Frameworks in Synthesis and Battery Applications. Adv Sci 8:2001980

\section{Figures}



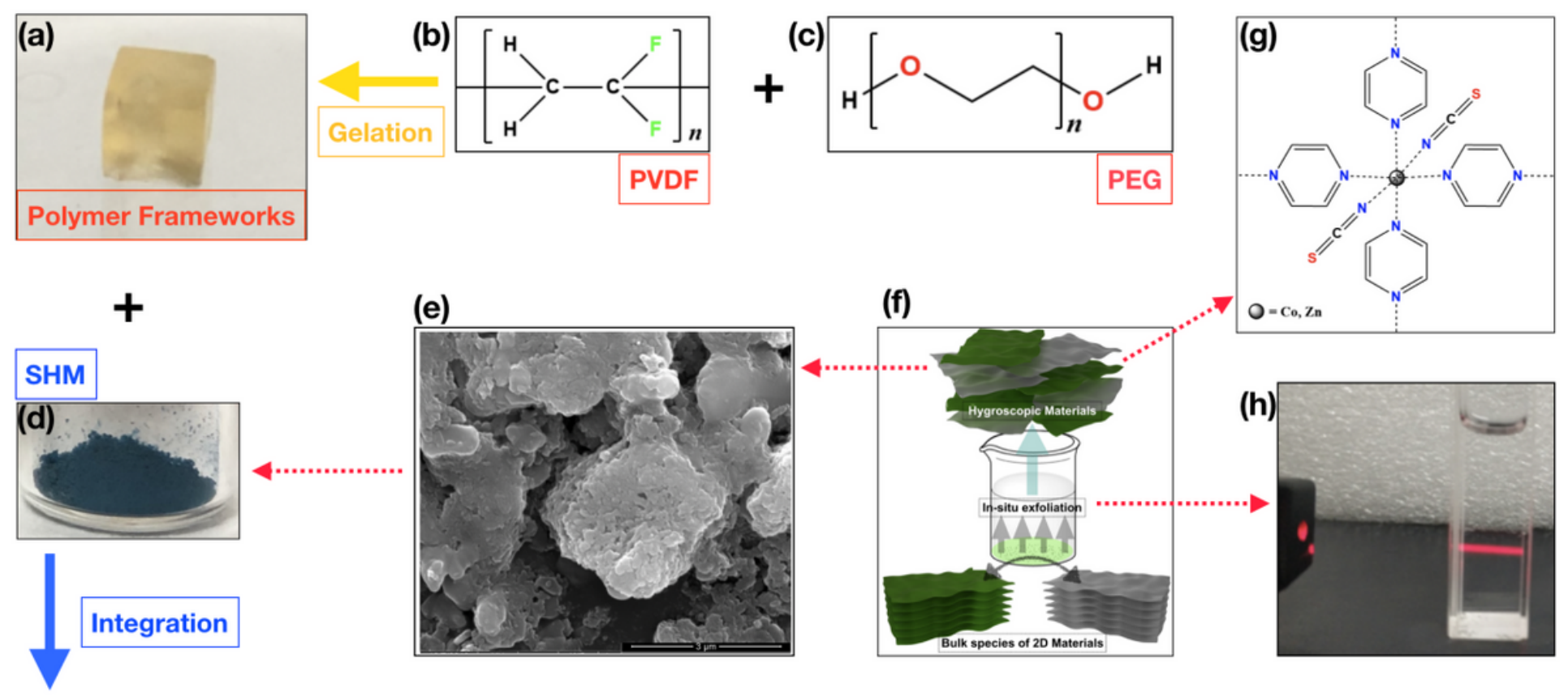

(i)

(j)

(k)
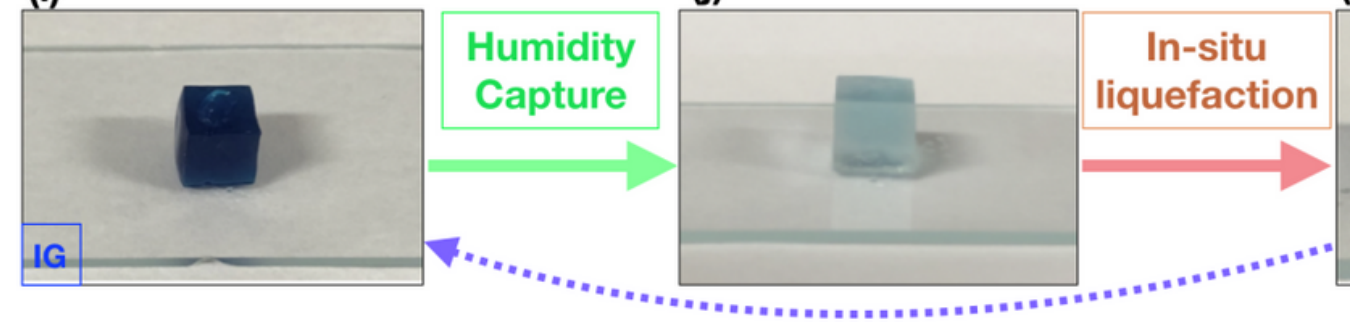

\section{Figure 1}

Preparation strategy and working principles of IG: (a)Photo image of blank gel that composed of (b) PVDF and (c) PEG; (d) photo image, (e) SEM image, (f) preparation method, (g) molecular structure, and (h) Tyndall effect of SHM; (i) photo image, (j) humidity capture, and (k) in-situ liquefaction of IG. 

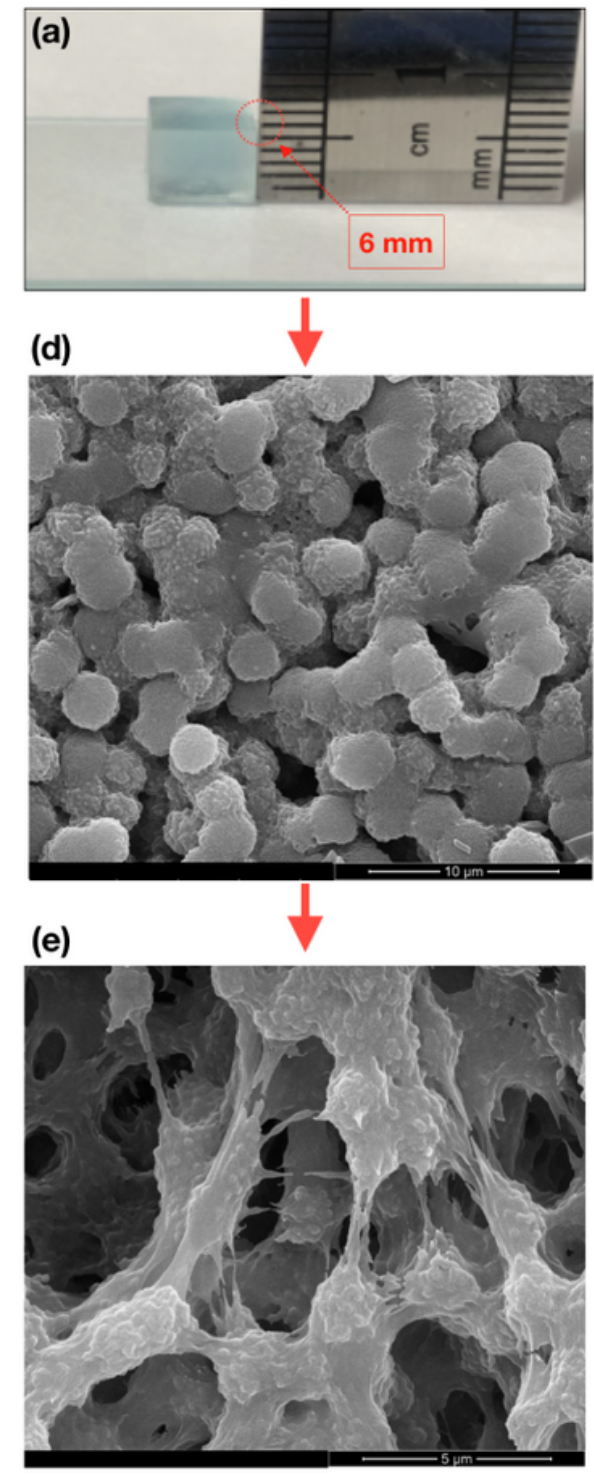
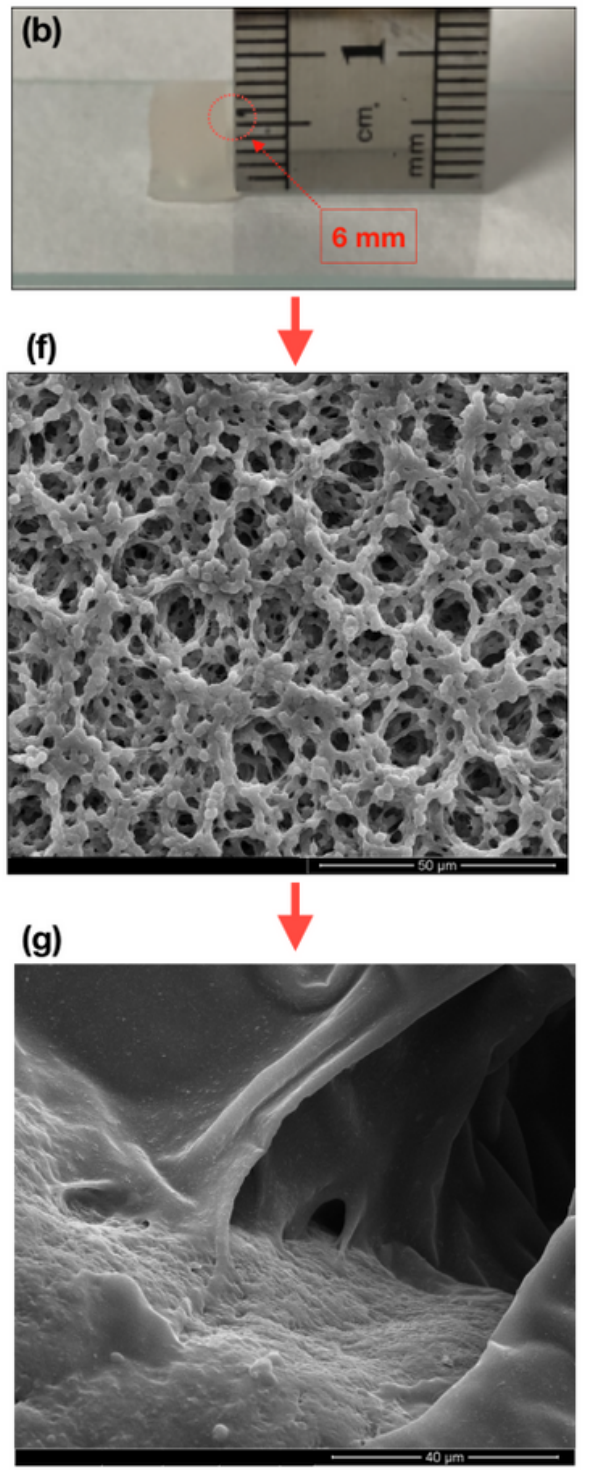
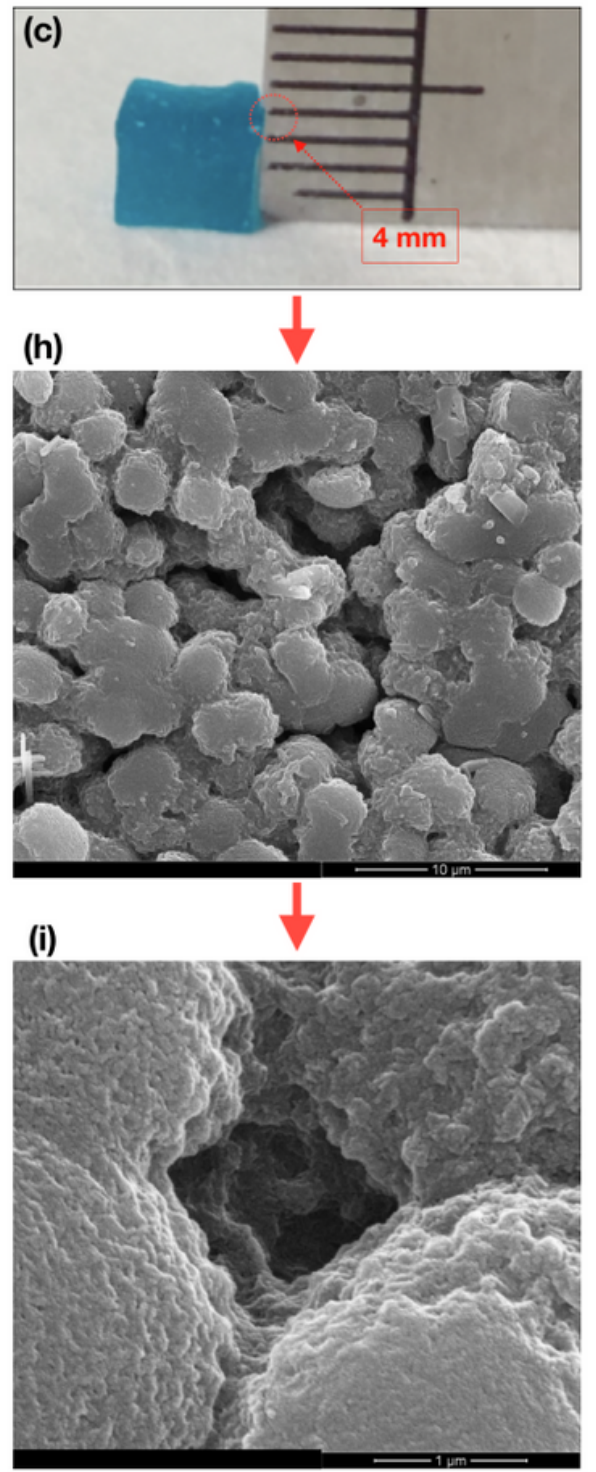

Figure 2

The photo (a-c) and SEM images (d-i) for IG (with weight ratio of SHM: PVDF = 30\%) under the state of $(a$, $d, e)$ as-prepared, $(b, f, g)$ after water adsorption, and (c, h, i) after water release. 


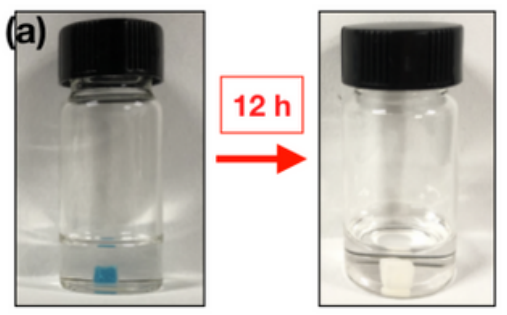

(b)

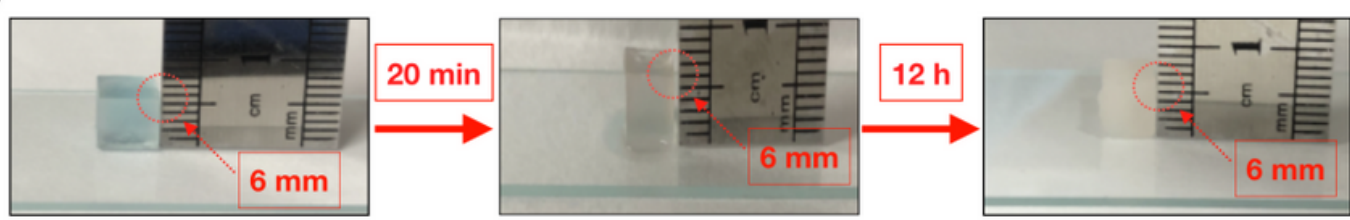

(c)
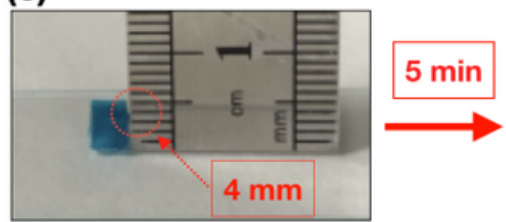

(d)

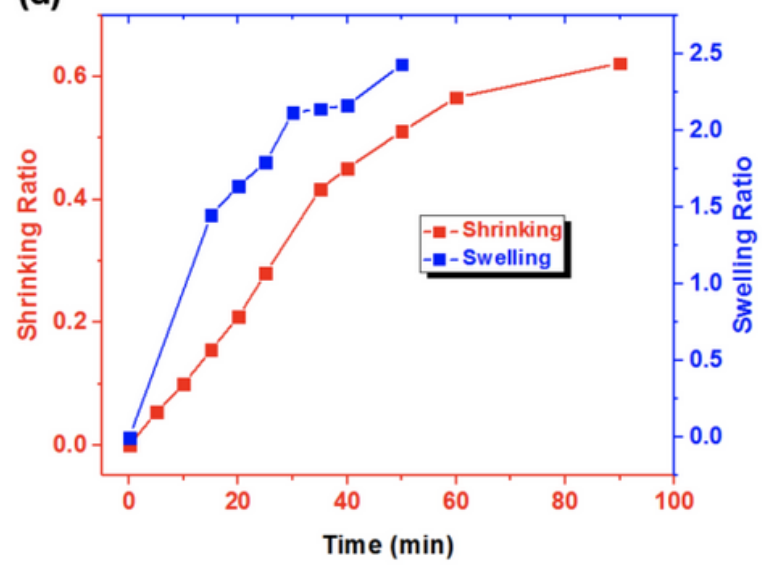

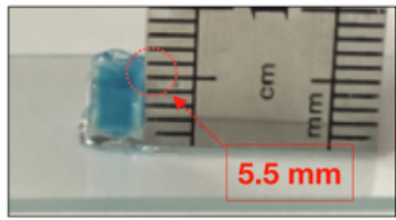

(e)

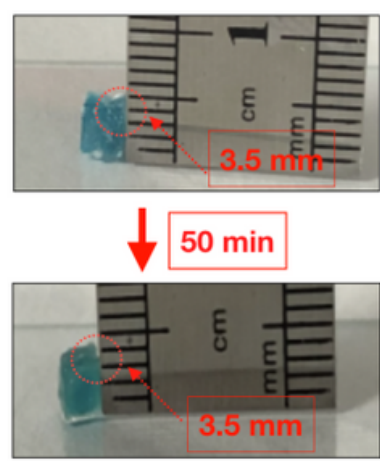

(f)

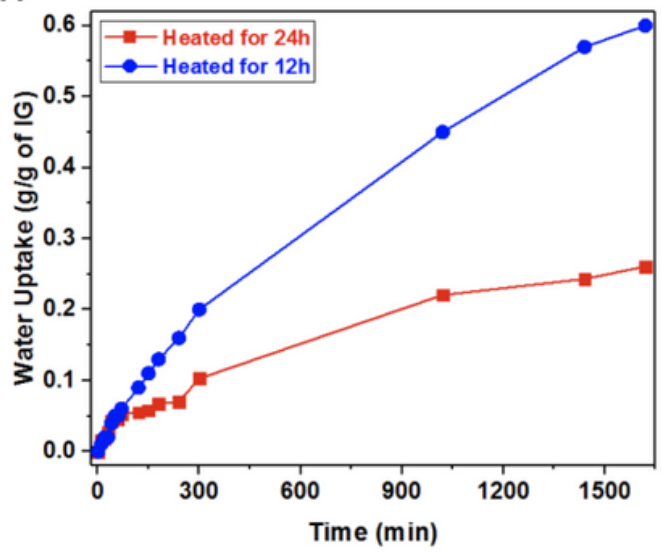

Figure 3

The time-dependent variation of $(\mathrm{a}, \mathrm{b})$ as-prepared gel immersed in water for $12 \mathrm{~h}$ and $(\mathrm{c})$ the shrinking gel (heated for $40 \mathrm{~min}$ ) immersed in DMF for $50 \mathrm{~min}$; (d) Comparison between shrinking and swelling ratio; (e) Variation of shrinking gel (heated for $2 \mathrm{~h}$ ) immersed in DMF for 50 min; (f) Comparison between the adsorption capacity of shrinking gel before and after destroying.

At this point, water release must lead to shrinking of the channels on IG, as expected, the diameter of the channel has decreased to be about $1 \mu \mathrm{m}$ (Figure $2 \mathrm{~h}$, i). Note again, this kind of 40 times shrinking in channels has only induced about 3.4 times decrease of the gel volume, further demonstrating the intelligence of the gel with superior adaptability to water. 


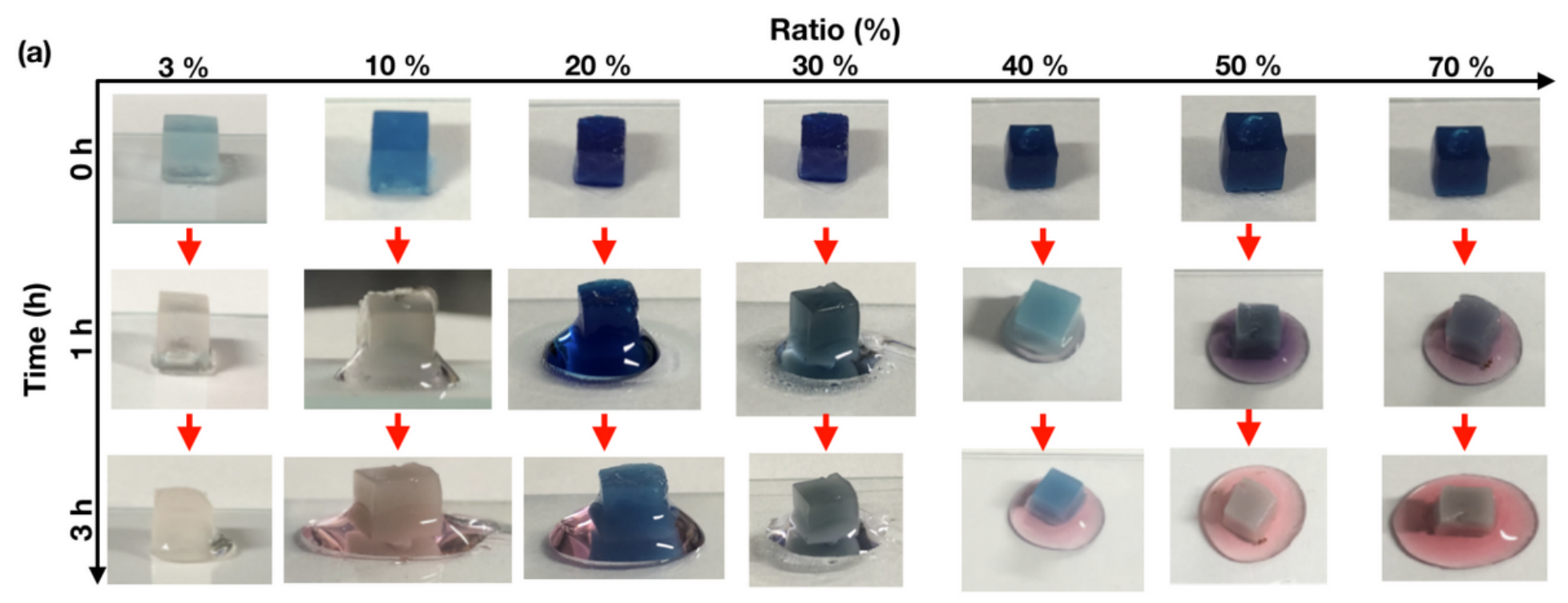

(b)

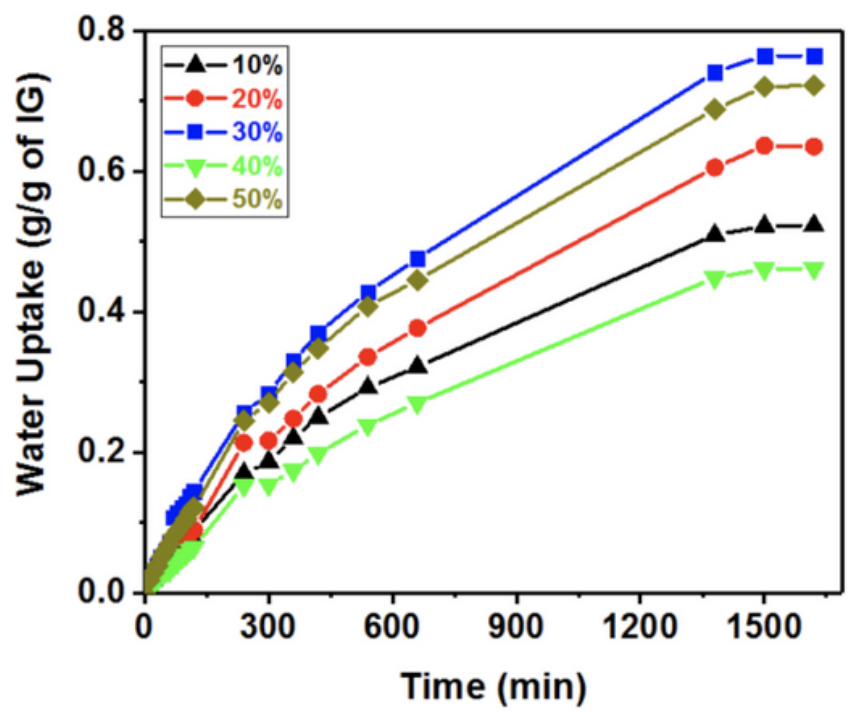

(c)

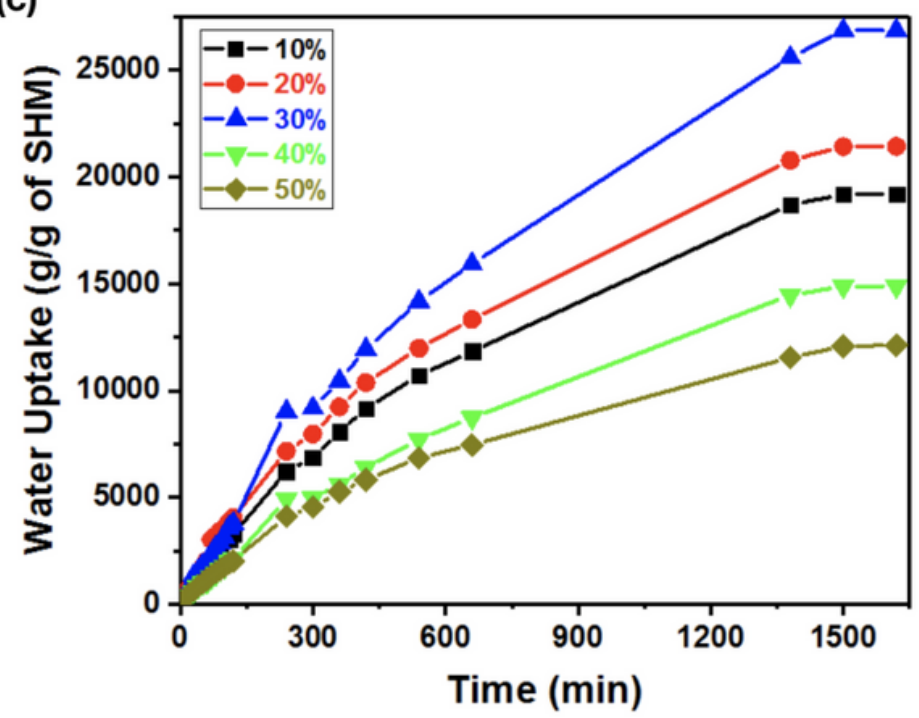

Figure 4

(a) Time-dependent variation of IG with different SHM:PVDF ratios upon exposure to ambient atmosphere; (b) Time-dependent water adsorption capacity based on the weight if IG; (c) Time-dependent water adsorption capacity based on the weight of SHM.

\section{Supplementary Files}

This is a list of supplementary files associated with this preprint. Click to download.

- SIECL.docx 